\title{
Instabilities of a columnar vortex in a stratified fluid
}

\author{
Patrice Meunier, Nicolas Boulanger, Xavier Riedinger and Stéphane Le Dizès
}

\begin{abstract}
In this paper, we show that the presence of a vertical density gradient can destabilise a Gaussian vortex in two different ways. First, when the vortex axis is slightly tilted with respect to the vertical and if its Froude number is larger than one, the vortex exhibits strong axial flows inside a critical layer, which lead to a violent three-dimensional instability. Second, when the vortex axis is vertical, the vortex is weakly unstable by emission of internal gravity waves. Theoretical predictions for both instabilities are compared with experimental evidences.
\end{abstract}

\section{Introduction}

It is well known that rotating and stratified turbulence contains many vortices whose axes tend to align with the background rotation axis and the density gradient direction. It is thus of interest to study the stability of such vortices to gain comprehension on the mechanisms which drive rotating and stratified turbulence. As a first step, we will focus here on the effect of stratification only.

There exist many inviscid instabilities which can destabilise a vortex in a homogeneous fluid. When the velocity profile of the vortex satisfies the Rayleigh criterion, the vortex is subject to a centrifugal instability. Otherwise, all the eigenmodes of the vortex (called vortex Kelvin modes) are neutral and can only grow due to an external forcing. For example, the presence of a second vortex can induce two different instabilities: the large wavelength Crow instability [5] and the short wavelength elliptical instability [9].

The presence of a stable stratification modifies the characteristics of these three instabilities. The Rayleigh criterion is not modified by stratification but the instability is weaker as soon as viscous effects are also considered [8]. The Crow instability

\section{Patrice Meunier}

Institut de Recherche sur les Phenomenes Hors Equilibre, CNRS / Aix-Marseille Universites, 49 rue Joliot-Curie, 13384 Marseille, France, e-mail: meunier@irphe.univ-mrs. fr 
is transformed into a zig-zag instability $[1 ; 6]$. And the elliptical instability is weakened due to the appearance of critical points in the vortex Kelvin modes [10].

We show in this paper that the presence of stratification can also destabilise a centrifugally stable vortex. After describing the vortex and the density profile in section 2, we first focus on the effect of a tilt of the vortex in section 3 . We then study the instabilities of a vertical vortex in section 4 and finally conclude in section 5 .

\section{A Gaussian vortex in a stratified fluid}

We wish to study the stability of a Gaussian vortex, whose angular velocity profile is defined as:

$$
\Omega(r)=\frac{v_{\theta}}{r}=\frac{\Gamma}{2 \pi r^{2}}\left(1-\mathrm{e}^{-r^{2} / a^{2}}\right)
$$

for a vortex of circulation $\Gamma$ and core size $a$. Such a vortex is stable with respect to the centrifugal instability in a non-stratified fluid. It is also interesting because it is the attractive solution for any localised patch of vorticity evolving due to viscous diffusion.

This vortex is placed in a vertical density stratification $\rho(z)$, which is assumed to be linear and characterized by a constant buoyancy frequency $N=\sqrt{-(g / \rho)(\partial \rho / \partial z)}$. We consider that the vortex axis may be tilted with respect to the vertical with an angle $\alpha$, as shown on Fig. 1(a).

This system is characterised by two non-dimensional parameters: the Froude number $F=\Gamma / 2 \pi N a^{2}$ and the Reynolds number $\operatorname{Re}=\Gamma / \mathrm{v}$.
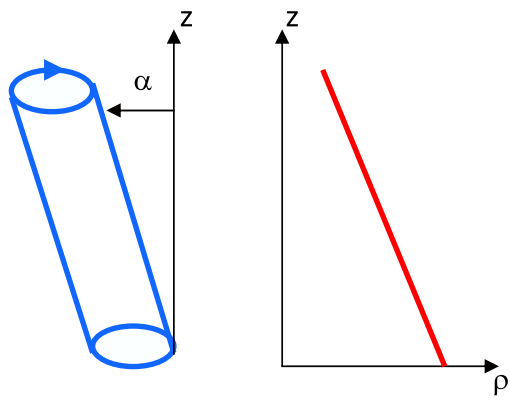

(a)

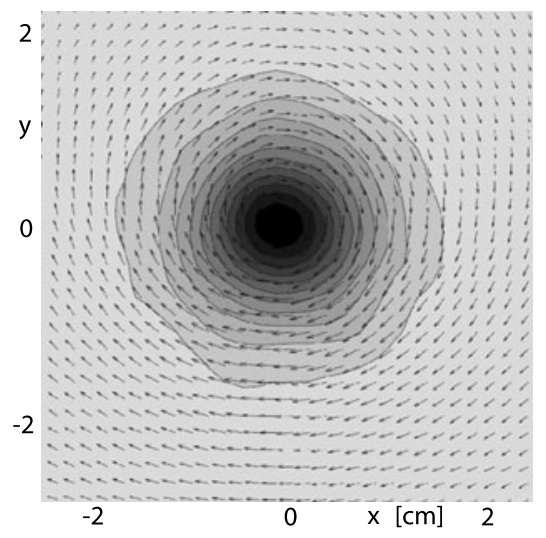

(b)

Fig. 1 (a) Sketch of a vortex inclined of an angle $\alpha$ with respect to a stable linear density stratfication $\rho(z)$. (b) Experimental velocity field (and vorticity contours) obtained for a vortex generated by a flat plate impulsively rotated in a stratified fluid measured by PIV. 
Experimentally, it is possible to create a Gaussian vortex by rotating a flap impulsively in a fluid initially at rest. Some PIV measurements (shown on Fig. 1b) allow to check that the vortex is circular and close to Gaussian. The linear stratification of the fluid is obtained using the two-tank method with salted water.

The linear temporal stability characteristics of the vertical vortex have also been obtained by a numerical spectral method.

\section{Instabilities of a tilted vortex}

We show in this section that the presence of a small tilt angle $\alpha$ can drastically change the structure and the stability of the vortex when its Froude number is larger than one.

\subsection{Spatial structure of a tilted vortex}

It has been shown [2] that tilting the vortex induces an axial velocity $w$ proportional to the tilt angle $\alpha$ for small angles:

$$
w=\alpha \frac{r \Omega(r)^{3}}{\Omega(r)-N^{2}} \sin \theta
$$

where $\theta$ is the angle with respect to the direction of the tilt. At low Froude number, the vertical velocity decreases as $1 / N^{2}$ and thus vanishes, leading to the same model of vortex described by [4], where the circular streamlines are horizontal but with their centers aligned on a tilted axis. However, when the Froude number is larger than 1 , this solution diverges at the critical radius $r_{c}$ where the angular velocity $\Omega\left(r_{c}\right)$ equals the buoyancy frequency $N$. This creates a critical layer with strong axial velocities.

Theoretically, the addition of the viscous terms smoothes the singularity in a viscous critical layer of width $R e^{-1 / 3}$. In this layer, the axial velocity reaches values of order $\alpha R e^{1 / 3}$. In Fig. 2, the analytic solution is compared to the experimental PIV measurement of the axial velocity. A good qualitative and quantitative agreement is observed. A cut along the direction of the tilt (x-axis) indicates that the velocity profile contains a strong positive jet on one side and a strong negative jet on the other side. On the contrary, the velocity profile in the direction perpendicular to the tilt (y-axis) exhibits two shear layers on each side of the vortex center. 


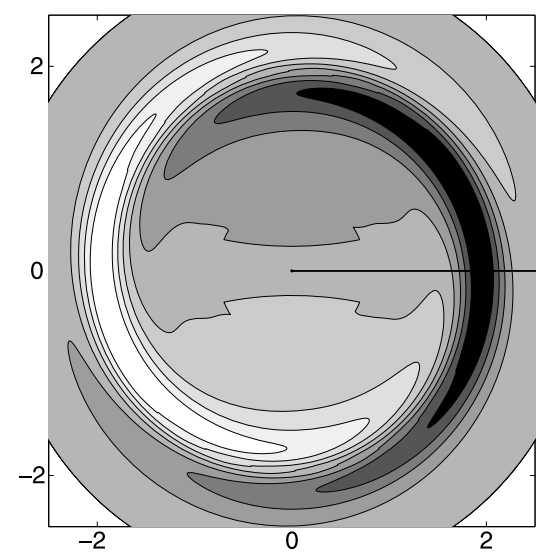

(a)

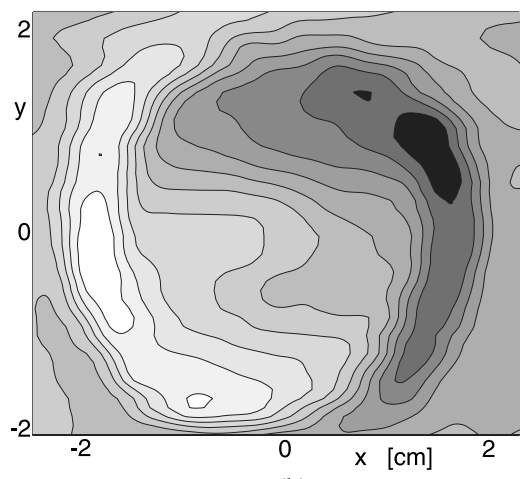

(b)

Fig. 2 (a) Theoretical and (b) experimental contours of axial velocity in a horizontal plane, for a vortex tilted with an angle $\alpha=0.07 \mathrm{rad} . \operatorname{Re}=450$ and $F=3.2$.

\subsection{Tilt-induced instabilities}

The presence of strong axial flows inside the critical layers can destabilise the vortex. Indeed, when the Reynolds number or the tilt angle is increased, the vortex suddenly exhibits strong three-dimensional perturbations which lead to a turbulent flow inside the vortex. Such perturbations are shown on the photographs of Fig. 3 in two different sideviews.

Fig. 3(a) shows a shadowgraph visualisation in the vertical longitudinal plane (i.e. the plane of the figure in Fig. 1), where the vortex thus appears tilted. The visualisation highlights a row of clockwise vortices on each side of the vortex. These vortices correspond to Kelvin-Helmholtz billows issued from a Kelvin-Helmholtz instability of the shear present in thes critical layer. In the tilted longitudinal plane (i.e. normal to the plane of the figure in Fig. 1), on the other hand, the perturbation seems to contain a row of alternate vortices on each side (see Fig. 3b), which is due to the sinuous mode of a jet instability.

These mechanisms are further confirmed by PIV measurements in these two planes shown in Fig. 4. In the vertical longitudinal plane (Fig. 4a), the jet instability made of alternate vortices is clearly visible on each side of the vortex axis. In the tilted longitudinal plane (Fig. 4b), each mixing layer destabilizes in a row of vortices. It can be noted that the different instabilities do not correspond between visualisations and PIV measurements: they are in phase quadrature. This is due to the fact that density structures are advected by the vortex and they are thus maximal at the end of the forcing by the flow, and not in the plane where the forcing is maximal. 


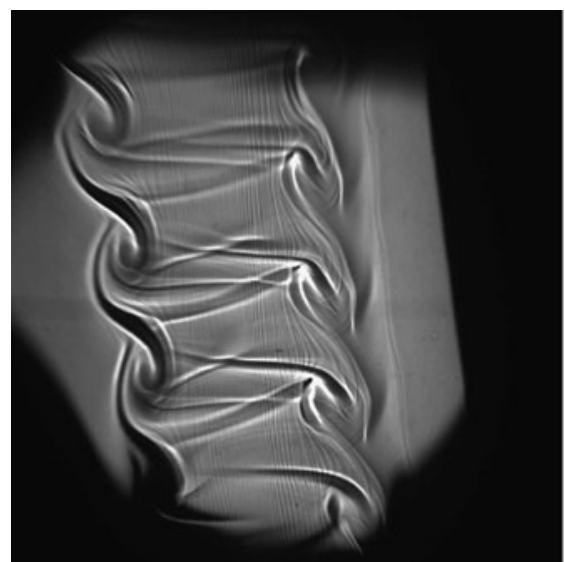

(a)

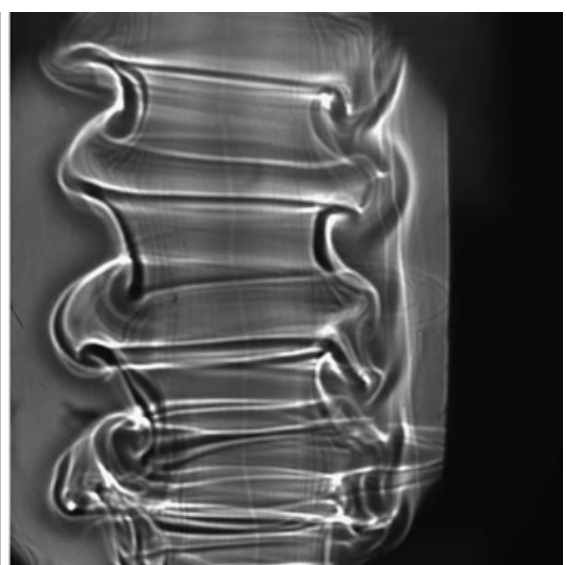

(b)

Fig. 3 Shadowgraph visualisation of the tilt-induced instabilities in (a) the vertical longitudinal plane and (b) the tilted longitudinal plane. $F=3, R e=720, \alpha=0.07 \mathrm{rad}$.

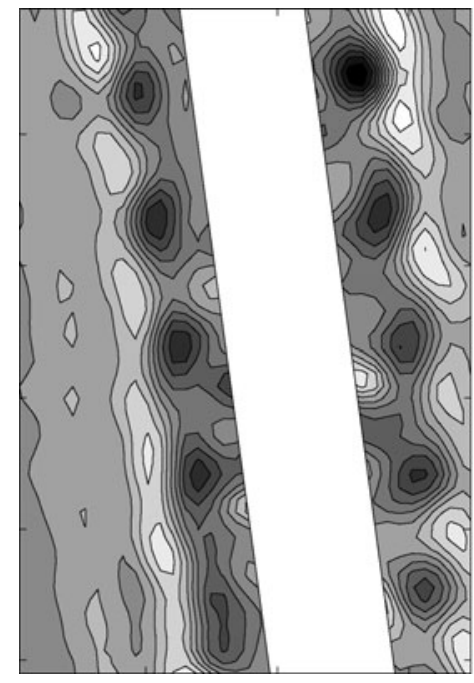

(a)

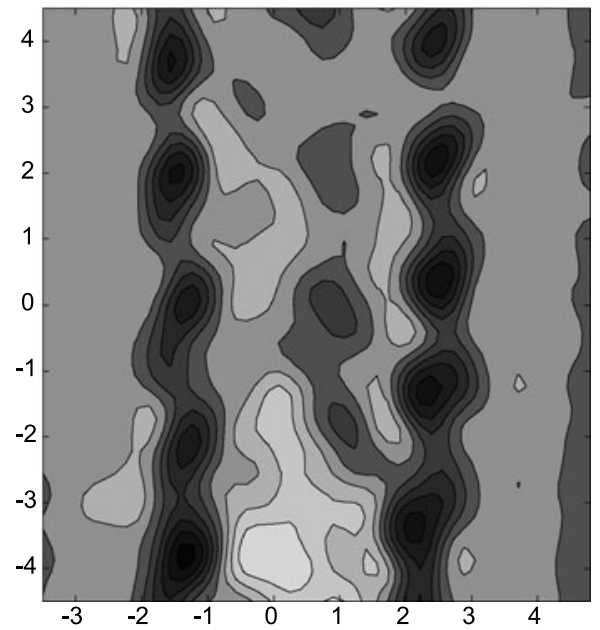

(b)

Fig. 4 PIV measurements of the instantaneous azimuthal vorticity showing the tilt-induced instabilities in (a) the vertical longitudinal plane and (b) the tilted longitudinal plane.

A local stability analysis has been performed [3] and proves that the threedimensional instability of the vortex is indeed linked to a local shear or jet instability inside the critical layer. The growth rate (of the order of $0.1 \alpha e^{2 / 3} N$ ) and the wavelength are in good quantitative agreement with the observations. Moreover, a 
qualitative criterion for the stability of a tilted stratified vortex can be obtained by saying that the growth rate must be larger than the angular frequency of the vortex. The vortex is thus unstable when $\alpha R e^{2 / 3} \gg 1$ and for $F>1$.

\subsection{Consequences}

These local instabilities create a strong turbulent flow inside the vortex. However, the vortex is not broken and its circulation remains constant even in the presence of instabilities. The only effect of the instabilities on the global properties of the vortex is to increase its core size $a$ if the Froude number $F$ is slightly larger than 1. This can be understood by the fact that for Froude of order 1, the critical layer is close to the vortex center and the mixing created by the instabilities can thus diffuse the vorticity more efficiently than for very large Froude number (for which the critical layer is far from the vortex center). In the presence of instabilities, the vortex core size increases, leading to a decrease of the Froude number until it becomes smaller than one at which the critical layer suddenly disappears.

Since the control parameter of the instability is $\alpha R e^{2 / 3}$, for the large Reynolds numbers of geophysical flow, vortices are expected to be unstable for very small tilt angle. This instability should create a deficit of vortices with a Froude number slightly above one, which could be interesting to analyse in oceanic and atmospheric data..

The strong axial velocity created in the critical layer may also be a visible effect. It could have an important impact on the diapycnal mixing in geophysical flows. It would be interesting to quantify its real contribution in oceans.

\section{Radiative instability}

We now focus on the stability of a Gaussian vortex whose axis is aligned with the density gradient $(\alpha=0)$.

\subsection{Linear stability analysis}

A linear stability analysis has been performed using a pseudo-spectral Chebyshev code. The boundary conditions, especially the condition of radiation at infinity, have been implemented by considering an integration in the complex plane. Further explanations on the numerical technique can be obtained in [12].

Figure 5(a) shows the growth rate of the most unstable eigenmode as functions of Froude and Reynolds numbers. Its spatial structure exhibits two opposite sign vortices at the center surrounded by weak edges spiralling around the vortex, associated 


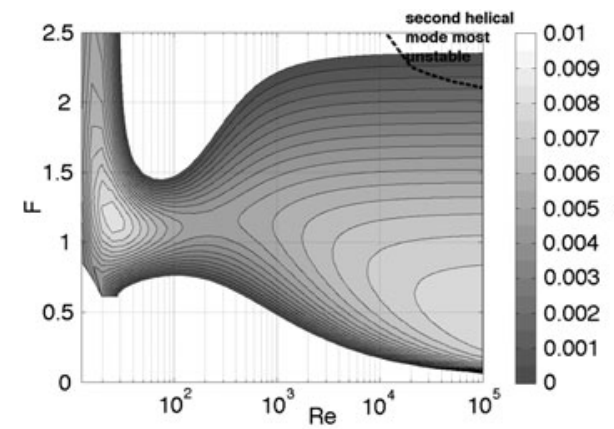

(a)

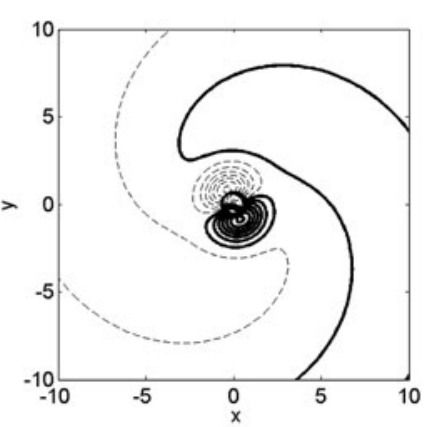

(b)

Fig. 5 (a) Growth rate of the most unstable mode for a vertical vortex in a stratified fluid. (b) Structure of the most unstable mode for $R e=10^{5}$ and $F=1.5$.

with internal gravity waves. This instablity is due to the gravity wave emission, as described in [7] and [11]. It can be understood as an interaction between the vortex Kelvin mode (creating the two central vortices) and internal gravity waves which radiate to infinity. Such a description has also been given in [13].

It can be noted that the vortex is unstable for any Reynolds number and is maximal for Froude numbers around 1. The damping of the instability at large Froude number is due to the appearance of a critical point in the eigenmode. The damping of the instability at small Froude numbers is due to viscous effects: the wavelength scales as $F$ for small Froude numbers, and the viscous damping rate $v k^{2}$ thus increases as $F^{-2}$ when $F$ tends to zero. These two effects explain why the instability is only present on a band of Froude numbers around one.

\subsection{Experimental evidence?}

Experiments have been performed to try to observe this instability. However, the growth rate of this instability is very small (close to $0.1 \%$ of the maximal angular velocity of the vortex) and the experimental results are not clear. The vortex center, visualised by a dye, usually exhibits an undulation as shown on Fig. 6 in the unstable region of the parameter space. The wavelength of the undulation is of the order of the most unstable wavelength found theoretically although it is usually larger. For the parameters of Fig. 6, the wavelength was measured to be twice larger than the predicted wavelength. 
Fig. 6 Dye visualisation of the instability of a vertical vortex in a stratified fluid (sideview). $F=1.15, R e=$ 610.

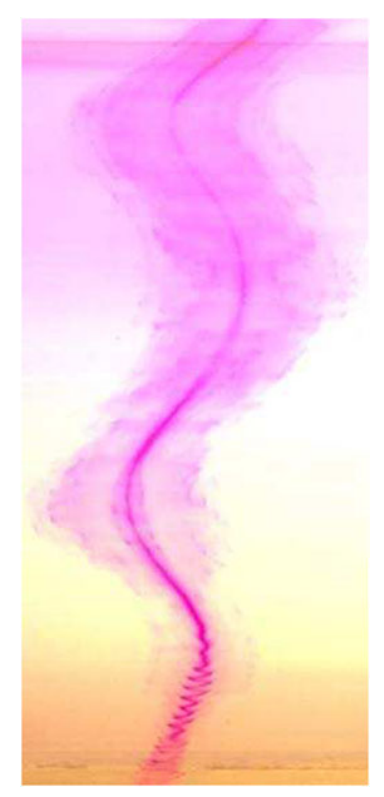

\section{Conclusion}

We have shown that the presence of stratification can drastically change the stability properties of a Gaussian vortex. Two cases have been studied. We have demonstrated that the presence of small tilt between the vortex axis and the density gradients can induce strong axial flows inside a critical layer, which lead to three-dimensional perturbations due to a Kelvin-Helmholtz or a jet instability. These instabilities are extremely violent and appear for very small tilt angles at large Reynolds numbers. Theoretical predictions are confirmed by experimental visualisations and measurements.

In a second study, we have shown theoretically that a vertical Gaussian vortex is unstable in a stably-stratified fluid whatever the Reynolds number in an interval of Froude number around one. The unstable mode creates an undulation of the vortex centerline and is associated with an emission of internal gravity waves far from the vortex center.

We believe that these results are interesting for geophysical applications because they show that the coherent structures found in stratified turbulence may not be stable, especially at high Reynolds numbers. Indeed, it is natural to think that vortices in a turbulent flow might experience a small tilt angle due to a small vertical shear or to the presence of weak internal waves. These vortices would thus be subject to the tilt-induced instability which is extremely powerful, even for small tilt angles. This would mean that stratified turbulence is not similar to a two-dimensional turbulence composed of horizontal vortices but that it is composed of intense three-dimensional motions at least for Froude numbers around 1. Another interesting consequence is 
that there is a coupling between vortices and internal gravity waves since vortices can emit internal gravity waves.

Acknowledgements The support of ACI grant 'Prévention des catastrophes naturelles' and ANR grant 'Fronts, secondary cyclones and gravity waves: instability, mixing and transport' by the french ministry of research is most gratefully acknowledged.

\section{References}

[1] Billant, P., Chomaz, J.M.: Experimental evidence for a new instability of a vertical columnar vortex pair in a strongly stratified fluid. J. Fluid Mech. 418, 167-188 (2000)

[2] Boulanger, N., Meunier, P., Le Dizès, S.: Structure of a stratified tilted vortex. J. Fluid Mech. 583, 443-458 (2007)

[3] Boulanger, N., Meunier, P., Le Dizès, S.: Tilt-induced instability of a stratified vortex. J. Fluid Mech. 596, 1-20 (2008)

[4] Canals, M., Pawlak, G., MacCready, P.: Tilted baroclinic tidal vortices. J. Phys. Oceanography 39(2), 333-350 (2009)

[5] Crow, S.C.: Stability theory for a pair of trailing vortices. AIAA J. 8(12), 2172-2179 (1970)

[6] Dritschel, D.G., Torre Juarez, M.1.: The instability and breakdown of tall columnar vortices in a quasi-geostrophic fluid. J. Fluid Mech. 328, 129-160 (1996)

[7] Ford, R.: The response of a rotating ellipse of uniform potential vorticity to gravity wave radiation. Phys. Fluids 6(11), 3694-3704 (1994)

[8] Hopfinger, E.J., van Heijst, G.J.F.: Vortices in rotating fluids. Annu. Rev. Fluid Mech. 25, 241-289 (1993)

[9] Kerswell, R.R.: Elliptical instability. Annu. Rev. Fluid Mech. 34, 83-113 (2002)

[10] Le Dizès, S.: Inviscid waves on a lamb-oseen vortex in a rotating stratified fluid: consequences for the elliptic instability. J. Fluid Mech. 597, 283-303 (2008)

[11] Plougonven, R., Zeitlin, V.: Internal gravity wave emission from a pancake vortex: an example of wave-vortex interaction in strongly stratified flows. Phys. Fluids 14(3), 1259-1268 (2002)

[12] Riedinger, X., Meunier, P., Le Dizès, S.: Viscous stability properties of a Lamb-Oseen vortex in a stratified fluid. J. Fluid Mech. 645, 255-278 (2010)

[13] Schecter, D.A., Montgomery, M.T., Reasor, P.D.: A theory for the vertical alignment of a quasigeostrophic vortex. J. Atm. Sciences 59(2), 150-168 (2002) 